\title{
Anti-MDA5 Antibody-positive Clinically Amyopathic Dermatomyositis Complicated by Unilateral Right-sided Interstitial Lung Disease
}

\author{
Mayuko Kori ${ }^{1}$, Nobuyasu Awano ${ }^{1}$, Minoru Inomata ${ }^{1}$, Naoyuki Kuse ${ }^{1}$, Mari Tone ${ }^{1}$, \\ Hanako Yoshimura ${ }^{1}$, Tatsunori Jo ${ }^{1}$, Kohei Takada ${ }^{1}$, Toshio Kumasaka ${ }^{2}$, \\ Tamiko Takemura ${ }^{2}$ and Takehiro Izumo ${ }^{1}$
}

\begin{abstract}
:
We herein report a case of anti-MDA5 antibody-positive, clinically amyopathic dermatomyositis complicated by unilateral interstitial lung disease (ILD) in a 78-year-old man with a history of left lung tumor resection. He was admitted due to a persistent fever and abnormal right pulmonary opacity. A transbronchial lung cryobiopsy revealed pulmonary fibrosis, and combined immunosuppressive therapy was initiated. Findings from multiple evaluations, including dynamic breathing magnetic resonance imaging, supported decreased perfusion, ventilation, and mobility of the left lung as etiological factors of unilateral lung ILD. When patients present with laterality of such findings, clinicians should be aware that atypical imaging findings may be observed.
\end{abstract}

Key words: clinically amyopathic dermatomyositis, anti-MDA5 antibody, unilateral interstitial lung disease, transbronchial lung cryobiopsy, dynamic breathing magnetic resonance imaging

(Intern Med 59: 401-408, 2020)

(DOI: 10.2169/internalmedicine.3604-19)

\section{Introduction}

Clinically amyopathic dermatomyositis (CADM) is a type of dermatomyositis (DM) that is characterized by typical cutaneous manifestations of DM with little or no muscle abnormalities and accounts for an estimated $5-20 \%$ of all cases of DM (1). Antibodies against melanoma differentiationassociated protein 5 (MDA5) mark this subtype of dermatomyositis, and it is reported to be associated with severe, progressive, and often fatal, interstitial lung disease (ILD) $(2,3)$.

Typical computed tomography (CT) findings of ILD in CADM include bilateral distribution of reticular opacities, volume loss, bronchiectasis, and infiltration (4). We herein report a rare case of anti-MDA5 antibody-positive CADM complicated by unilateral ILD in a patient with a history of tumor resection in the left lung.

\section{Case Report}

A 78-year-old man with a history of left upper lobe lung cancer (pT2aN2M0, stage IIIA) and consequent left upper lobectomy had been followed for 5 years with no recurrence on CT imaging. He had been initially admitted to the Department of Urology in January 20XX for bladder cancer surgery and underwent three transurethral bladder tumor resection (TURBT) procedures. After the third procedure in late March, 20XX (day 1), he developed a persistent fever of unknown origin (body temperature more than $38^{\circ} \mathrm{C}$ ) that was refractory to meropenem hydrate. $\mathrm{He}$ also presented with chronic cough for a few months and subacute, worsening dyspnea on exertion. In addition, an abnormal opacity in the chest region noted on $\mathrm{CT}$ imaging appeared to be pro-

${ }^{1}$ Department of Respiratory Medicine, Japanese Red Cross Medical Center, Japan and ${ }^{2}$ Department of Pathology, Japanese Red Cross Medical Center, Japan

Received for publication July 2, 2019; Accepted for publication August 8, 2019

Correspondence to Dr. Nobuyasu Awano, awanobu0606@hotmail.co.jp 

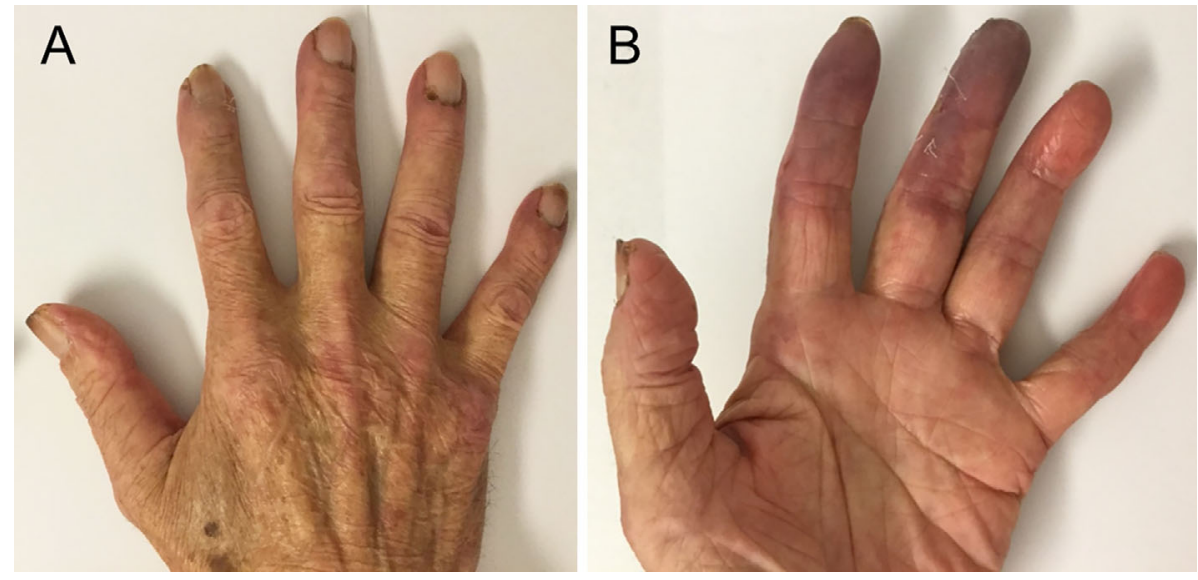

Figure 1. Physical findings. (A) Skin lesion on the right hand including Gottron's papule and peripheral atrophy. (B) Cyanosis of the fingers of the left hand.

gressing, and he was referred to our department on day 35 , for a further investigation and management.

His medical history included chronic obstructive pulmonary disease, type 2 diabetes mellitus, and hypertension. He was an ex-smoker (52 pack years until he quit at 72 years old) and had been chronically exposed to asbestos (between 40-65 years old). His regular medications were miglitol, sitagliptin phosphate, and telmisartan.

On admission to our service, vital signs were normal except for a slight fever of $37.6^{\circ} \mathrm{C}$ and an oxygen saturation of $100 \%$ with $2 \mathrm{~L} / \mathrm{min}$ oxygen via nasal cannula. A physical examination revealed fine crackles in the middle to lower lung fields at the back of the right lung along with decreased breath sounds in the left lung. On inspection, movement of the left chest wall had decreased more severely than that of the right chest wall. The patient had skin lesions and symptoms characteristic of dermatomyositis, namely Gottron's papule, dorsal dermal sclerosis, peripheral cyanosis, ulcerations and peripheral atrophy of fingers, erythema of the nail beds (periungual erythema; Fig. 1), Raynaud's phenomenon, and morning stiffness. He did not complain of muscle weakness or myalgia, and manual muscle testing of the extremities was normal.

The laboratory findings are shown in Table 1 and were significant for elevated levels of aldolase (8.3 IU/L), ferritin (253 ng/mL), Krebs von den Lungen (KL-6) (1,133 U/mL), and inflammatory markers-namely $\mathrm{C}$-reactive protein $(9.16$ $\mathrm{mg} / \mathrm{dL})$ and $\mathrm{WBC}(10,220 / \mu \mathrm{L})$; however, the creatine kinase level was normal. An arterial blood gas analysis was remarkable only for increased $\mathrm{A}-\mathrm{aDO}_{2}$ (21 Torr, room air). Immunological investigations were positive for anti-nuclear antibodies, notably a cytoplasmic pattern (1:40), along with anti-MDA5 antibody (titer: 189 index value; normally $<32.0$, determined using an enzyme-linked immunosorbent assay; MEASACUP anti-MDA5 test, Corona Electric, Hitachinaka, Japan). Pulmonary function tests demonstrated restriction and diffusion impairment.

Chest X-ray acquired immediately after transfer to our department revealed unilateral ground-glass opacity and reticu- lation in the right middle and lower lung fields (Fig. 2A). Subsequent high-resolution CT of the right lung showed prevailing basilar reticulation associated with a decreased volume and bronchiectasis in the lower lobe, whereas no abnormalities were noted in the left lung (Fig. 2B, C). Lung ventilation scintigraphy demonstrated laterality (right:left = 61.6\%:38.4\%) consistent with lobectomy (Fig. 3). Dynamic breathing magnetic resonance imaging (MRI) was performed at a fixed mid-coronal plane through the trachea and at two fixed mid-sagittal planes through the middle of each lung, as previously described. The patient was asked to breathe slowly and deeply from maximal inspiration to maximal expiration several times (5). Thoracic movement was evaluated by measuring the dimensions of the chest wall in three regions in cine images: longitudinal thoraces (from the apex to diaphragm) on the mid-coronal plane and upper and lower anteroposterior thoraces $(5 \mathrm{~cm}$ below the apex and 5 $\mathrm{cm}$ above the diaphragm, respectively, at maximal inspiration) on the mid-sagittal planes. The anteroposterior dimension of the thorax (ADT) and longitudinal dimension of the thorax (LDT) were measured at maximum inspiration and maximum expiration as follows: a, LDT at maximal inspiration; b, LDT at maximal expiration; c, ADT of the upper thoraces at maximal inspiration; d, ADT of the upper thoraces at maximal expiration; e, ADT of the lower thoraces at maximal inspiration; and $\mathrm{f}$, ADT of the lower thoraces at maximal expiration. The mobility of the thoraces was evaluated based on the amplitude of respiratory motions and calculated as the difference between LDT and ADT at maximal inspiration and expiration (LDT: a-b, upper ADT: c-d, lower ADT: e-f). Our patient showed relative impairment of mobility and a reduced amplitude of respiratory motion in the left lung on both the coronal and sagittal planes (Fig. 4, Table 2).

He underwent bronchoscopy on day 35, for a further investigation, and bronchoalveolar lavage (BAL) of the middle lobe of the right lung was performed. The total cell number in the BAL fluid was $3.7 \times 10^{5} / \mathrm{mL}$, and cytology was negative (class I). A fluid analysis showed an elevated lympho- 
Table 1. Laboratory Test Results.

\begin{tabular}{|c|c|c|c|}
\hline \multicolumn{2}{|c|}{ Complete blood count } & \multicolumn{2}{|l|}{ Immunology } \\
\hline WBC & $10,220 / \mu \mathrm{L}$ & $\mathrm{RF}$ & $9 \mathrm{IU} / \mathrm{mL}$ \\
\hline $\mathrm{Hb}$ & $9.8 \mathrm{~g} / \mathrm{dL}$ & Anti-CCP antibody & - \\
\hline \multirow[t]{2}{*}{ Plt } & $347 \times 10^{3} / \mathrm{L}$ & Anti-nuclear antibody & $1: 40$ \\
\hline & & NUCLEOLAR & $1: 40$ \\
\hline \multicolumn{2}{|l|}{ Blood Chemistry } & CYTOPLASMIC & $1: 40$ \\
\hline AST & $61 \mathrm{IU} / \mathrm{L}$ & Anti-Jo-1 antibody & - \\
\hline ALT & $22 \mathrm{IU} / \mathrm{L}$ & Anti-U1-RNP antibody & - \\
\hline LDH & $335 \mathrm{IU} / \mathrm{L}$ & Anti-Sm antibody & - \\
\hline $\mathrm{CK}$ & $82 \mathrm{U} / \mathrm{L}$ & Anti-SS-A antibody & - \\
\hline Aldolase & $8.3 \mathrm{IU} / \mathrm{L}$ & Anti-SS-B antibody & - \\
\hline $\mathrm{TP}$ & $6.5 \mathrm{~g} / \mathrm{dL}$ & Anti-ds-DNA antibody & - \\
\hline Albumin & $2.7 \mathrm{~g} / \mathrm{dL}$ & Anti-Scl-70 antibody & - \\
\hline $\mathrm{Na}$ & $132 \mathrm{mEq} / \mathrm{L}$ & PR3-ANCA & - \\
\hline K & $4.4 \mathrm{mEq} / \mathrm{L}$ & MPO-ANCA & - \\
\hline BUN & $14 \mathrm{mg} / \mathrm{dL}$ & Anti-centromere antibody & - \\
\hline Cre & $1.19 \mathrm{mg} / \mathrm{dL}$ & Anti-RNA polymerase III antibody & - \\
\hline CRP & $9.16 \mathrm{mg} / \mathrm{dL}$ & Anti-ARS antibody & - \\
\hline $\operatorname{ESR}(1 \mathrm{~h})$ & $82 \mathrm{~mm}$ & Anti-Mi-2 antibody & - \\
\hline KL-6 & $1,133 \mathrm{U} / \mathrm{mL}$ & Anti-Ku antibody & - \\
\hline SP-D & $33.2 \mathrm{ng} / \mathrm{mL}$ & Anti-PM-Scl100 antibody & - \\
\hline \multirow[t]{2}{*}{ Ferritin } & $253 \mathrm{ng} / \mathrm{mL}$ & Anti-SRP antibody & - \\
\hline & & Anti-Jo-1 antibody & - \\
\hline \multicolumn{2}{|c|}{ Arterial blood gas analysis (room air) } & Anti-PL-7 antibody & - \\
\hline $\mathrm{pH}$ & 7.38 & Anti-PL-12 antibody & - \\
\hline $\mathrm{PaCO}_{2}$ & 38 Torr & Anti-OJ antibody & - \\
\hline $\mathrm{PaO}_{2}$ & 82 Torr & Anti-EJ antibody & - \\
\hline $\mathrm{HCO}_{3}^{-}$ & $24 \mathrm{mmol} / \mathrm{L}$ & Anti-Ro-52 antibody & - \\
\hline \multirow[t]{2}{*}{$\mathrm{A}-\mathrm{aDO}_{2}$} & 21 Torr & Anti-TIF1- $\gamma$ antibody & - \\
\hline & & Anti-MDA-5 antibody & 189 \\
\hline
\end{tabular}

cyte count $(37.2 \%)$; in addition, neutrophils (18.4\%), eosinophils $(4.8 \%)$, and macrophages $(39.6 \%)$ were observed. No microorganisms were cultured from the BAL fluid. Two biopsy samples, $20 \times 8 \mathrm{~mm}^{2}$ in size, were obtained from the right lung $\left(\mathrm{B}^{9} \mathrm{a}, \mathrm{B}^{9} \mathrm{~b}\right)$ through a transbronchial lung cryobiopsy (TBLC). The pathological evaluation of the biopsy tissue revealed thickening and edematous detachment of the alveolar wall, a collapsed alveolar space with cellular infiltration (macrophages and lymphocytes), and increased elastic fibers. Although pathological findings suggestive of diffuse alveolar damage (DAD), such as hyaline membrane and extensive fibrin, were not detected, fibrosis was remarkable in the interstitial tissue as well as in the lumen of the collapsed alveolae (Fig. 5). Asbestos bodies were not detected. A skin biopsy from the left dorsal hand revealed edematous detachment of the dermo-epidermal junction and lymphocytic infiltration of the dermis. Based on the above findings, a diagnosis of anti-MDA5 antibody-positive clinically amyopathic dermatomyositis (CADM) associated with ILD was made $(6,7)$. Physical and laboratory findings were negative for other comorbid connective tissue diseases.
The clinical course of the patient is depicted in Fig. 6. Given the possibility of his condition being the early phase of rapidly progressive ILD (RP-ILD), he was treated with combination therapy from day 43, after his diagnosis, which comprised oral prednisolone at $50 \mathrm{mg} /$ day and oral tacrolimus at $3 \mathrm{mg} /$ day. Intravenous cyclophosphamide (IVCY) $500 \mathrm{mg}$ was administered every 21 days, beginning on day 49. Prednisolone was tapered to $30 \mathrm{mg}$ /day after 2 weeks, and the tacrolimus dosage was increased to $5 \mathrm{mg} /$ day, with therapeutic drug monitoring. These treatments led to a gradual reduction in KL-6 and ferritin. However, there was a slight increase in the oxygen demand, which was consistent with our observation of slowly worsening reticulation on chest CT. The patient was discharged from the hospital on day 83 , with home oxygen therapy. In total, the patient was administered IVCY four times, and prednisolone was gradually reduced to $20 \mathrm{mg} /$ day. His respiratory condition remained stable thereafter, and he remained alive for the next six months. 


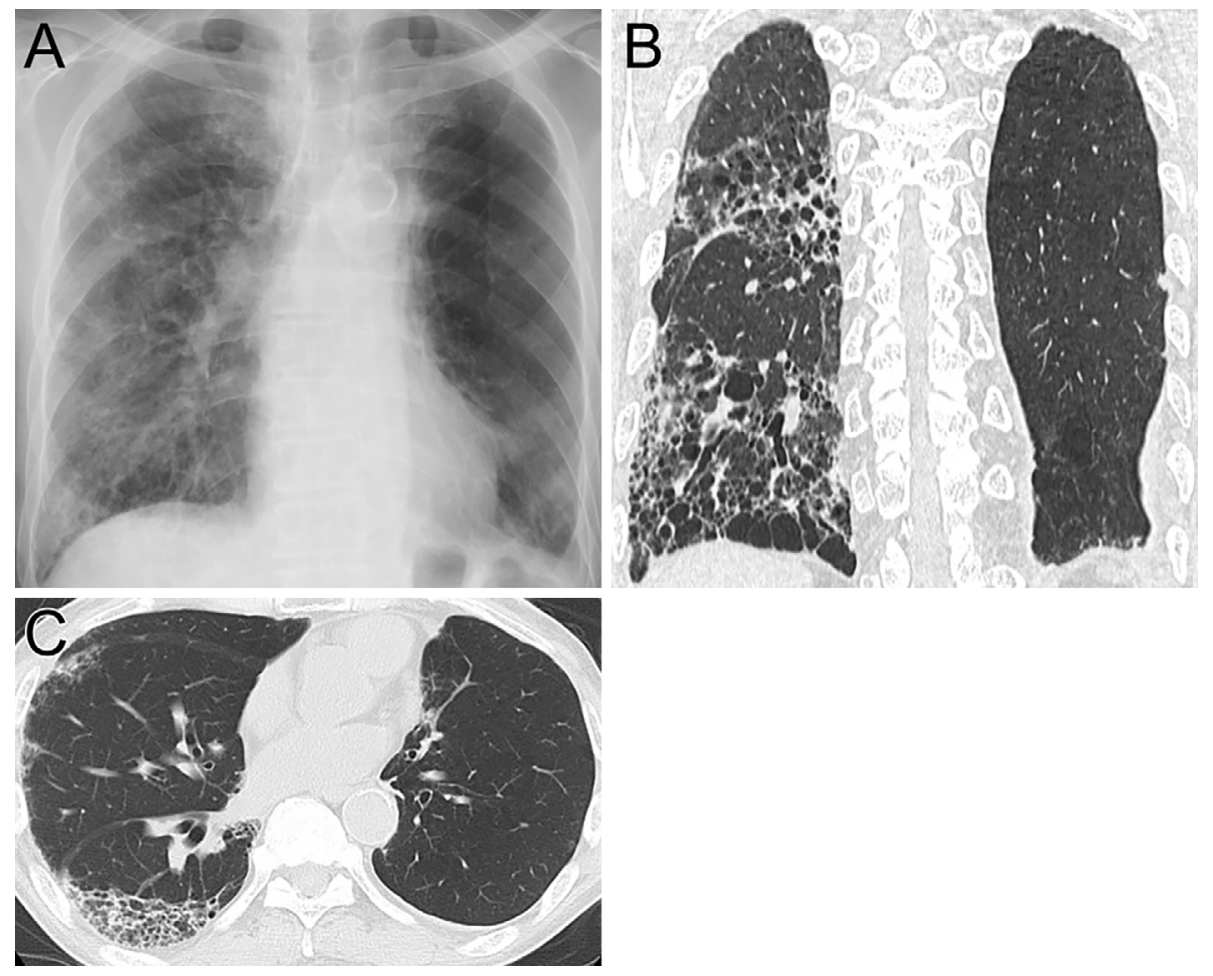

Figure 2. Radiological findings. (A) Chest X-ray showing ground-glass opacity and reticular consolidation, predominantly in the right middle and lower lung fields. (B, C) High-resolution computed tomography of the chest. Prevailing basilar reticulation with a decreased volume and bronchiectasis is observed in the right lung, whereas few remarkable findings are seen in the remaining lobes of the left lung.
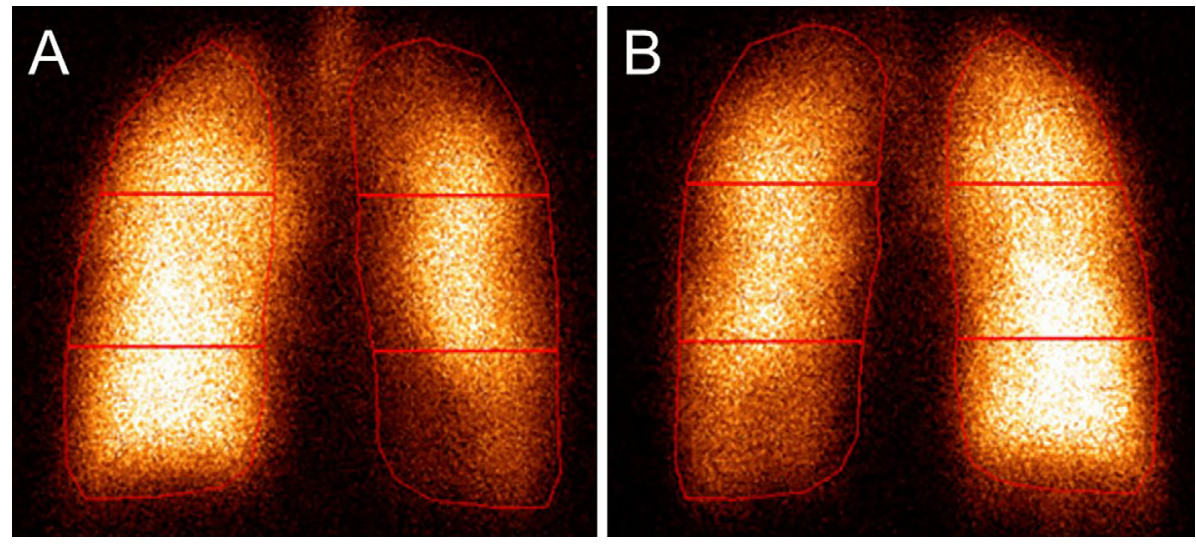

Figure 3. Lung ventilation scintigraphy (A and B) demonstrating laterality (right: left=61.6\%: $38.4 \%$ ).

\section{Discussion}

We encountered a case of anti-MDA5 antibody-positive CADM complicated by unilateral ILD and displaying atypical imaging features. CADM is associated with a poor prognosis due to the development of RP-ILD, especially when patients test positive for anti-MDA5 antibody $(8,9)$. In our patient, the pathological features indicated remarkable damage to the alveoli, suggesting possible progress to DAD. Furthermore, a non-DAD pattern in RP-ILD with anti-
MDA5 antibody-positive CADM has been reported; in this pattern, the pathological findings show diffuse and homogeneous inflammatory cell infiltration without a DAD pattern. The pathological pattern was diagnosed as unclassifiable interstitial pneumonia and inferred to be the early phase of RP-ILD (10). The pathological findings of our patient also indicated a non-DAD pattern. However, given the above facts and subacute clinical course of the respiratory symptoms and radiological findings, our patient was likely to develop RP-ILD. Therefore, we administered triple combined immunosuppressive therapy, which resulted in a survival for 

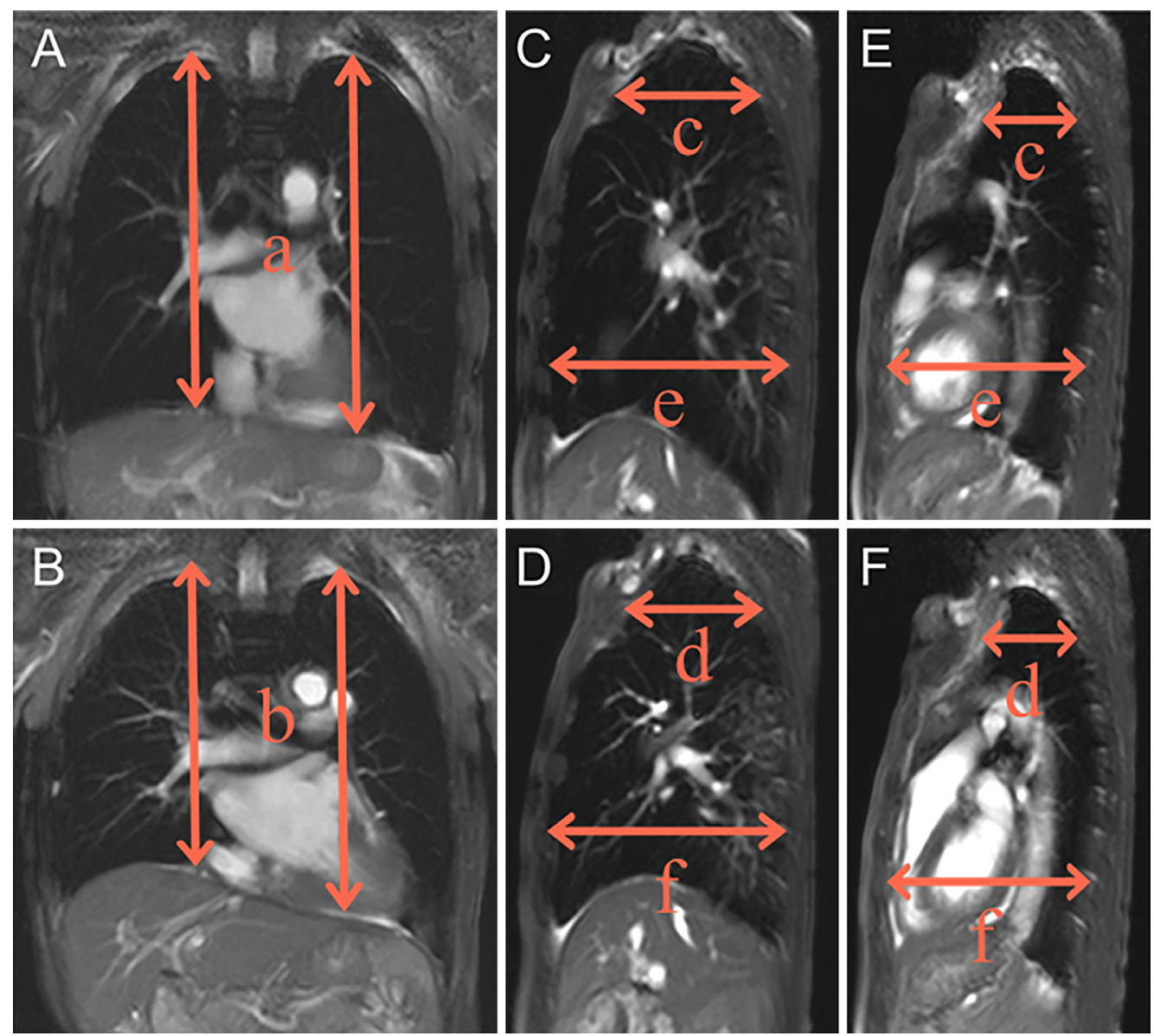

Figure 4. Imaging findings. (A-F) Dynamic breathing magnetic resonance imaging at a fixed midcoronal plane through the trachea and at two fixed mid-sagittal planes through the center of each lung. The patient was asked to breathe slowly and deeply from maximal inspiration to maximal expiration several times. Thoracic movement was evaluated using cine images by measuring the dimensions of the chest wall in three regions: the upper and lower anteroposterior thoraces $(5 \mathrm{~cm}$ below the apex and $5 \mathrm{~cm}$ above the diaphragm, respectively, at maximal inspiration) on mid-sagittal planes and longitudinal thoraces (from the apex to the diaphragm) on mid-coronal planes (A: maximal inspiration diaphragmatic motion, B: maximal expiration diaphragmatic motion, C: maximal inspiration chest wall motion of the right thorax, D: maximal expiration chest wall motion of the right thorax, $\mathrm{E}$ : maximal inspiration chest wall motion of the left thorax, and F: maximal expiration chest wall motion of left thorax). The anteroposterior dimension of the thorax (ADT) and longitudinal dimension of the thorax (LDT) were measured in each thorax at maximum inspiration and maximum expiration. As shown in Table 2, conspicuous laterality in the chest wall movement was observed. ADT: anteroposterior dimension of the thorax, LDT: longitudinal dimension of the thorax

Table 2. Dynamic Breathing Magnetic Resonance Imaging.

\begin{tabular}{|c|c|c|c|c|}
\hline & & Maximum inspiration & Maximum expiration & Amplitude of respiratory motions \\
\hline \multirow{3}{*}{ LDT, cm } & & (a) & (b) & (a) $-(b)$ \\
\hline & right & 222.6 & 179.7 & 42.9 \\
\hline & left & 238.6 & 213.8 & 24.8 \\
\hline \multirow{3}{*}{ ADT (upper), $\mathrm{cm}$} & & (c) & (d) & $(\mathrm{c})-(\mathrm{d})$ \\
\hline & right & 104.3 & 94.3 & 10 \\
\hline & left & 85.4 & 83.1 & 2.3 \\
\hline \multirow{3}{*}{ ADT (lower), cm } & & (e) & (f) & $(e)-(f)$ \\
\hline & right & 147.3 & 134.3 & 13 \\
\hline & left & 132.8 & 130.8 & 2 \\
\hline
\end{tabular}

LDT: longitudinal dimension of the thorax (from the apex to diaphragm), ADT: anteroposterior dimension of the thorax (anteroposterior diameter of the chest walls) 


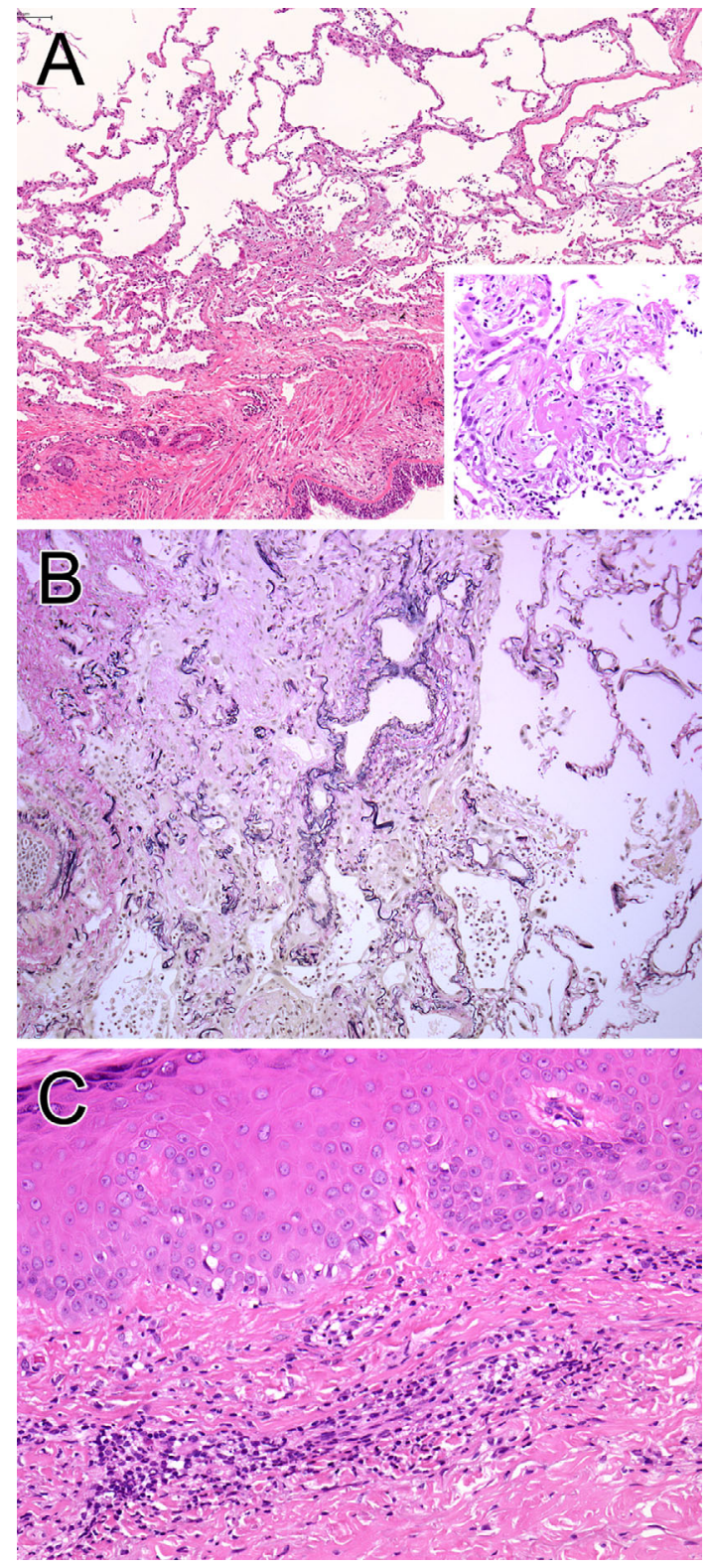

Figure 5. (A) The alveolar walls surrounding the bronchus were thickened due to fibrosis and/or edema, with mild lymphocyte infiltration and type II pneumocyte proliferation, indicating cellular alveolitis. Some intra-alveolar organization in the alveolar spaces is present $(\mathrm{A}$, inset). (B) The peribronchial alveoli collapsed with myxoid stroma to form peribronchial fibrosis. (C) A skin biopsy demonstrated basement membrane degeneration and perivascular lymphocyte infiltration.

six months. This case illustrates the possible improvement in the prognosis of CADM after a rapid diagnosis and treatment.

A TBLC has recently been deemed both sufficient and safe for establishing a histopathological diagnosis of ILD (11), as it provides sufficiently large samples that enable an accurate diagnosis, thereby leading to the prompt administration of appropriate treatment. Furthermore, previous studies using cryobiopsies have reported fewer complications than with a conventional surgical lung biopsy. In the present case, specimens of satisfactory size were obtained without any adverse events, demonstrating the efficacy of a TBLC.

Our patient had asymmetrical interstitial pneumonia, which is a rare presentation associated with connective tissue disease-associated ILD (CTD-ILD). Several possible causes of unilateral interstitial pneumonia have been previously reported, including gastroesophageal reflux (GER), vascular perfusion abnormalities of the lung, abnormal pulmonary ventilation, and impaired mobility of the thorax (12-15). Therefore, we examined if and how these factors might have contributed to the etiology. Our patient did not report symptoms typical of GER, and it has been reported that patients with GER do not always present with gastrointestinal symptoms. We did not perform specialized studies such as $\mathrm{pH}$ impedance monitoring or esophageal manometry; however, upper gastrointestinal endoscopy showed no abnormalities of the gastrointestinal mucosa. We therefore concluded that GER was not a likely etiological factor. In addition, a relatively low perfusion of the left lung was expected in our patient due to vascular resection during left upper lobectomy. In cases of idiopathic pulmonary fibrosis, increased blood flow is known to induce fibroblasts, which in turn leads to fibrosis, and the imaging findings in the present case are consistent with such a scenario (16). However, we were unable to clearly detect a left vs. right difference in the lung perfusion, as lung perfusion scintigraphy was not available in our facility. Next, we assessed the pulmonary ventilation using scintigraphy and found a reduced ventilation of the left lung compared with the right. Several studies have reported that elevated superoxide and hydrogen peroxide levels following hyperoxia cause lung injury (17-20). It is thus possible that the relative increase in the ventilation in the right lung led to hyperoxia and eventual fibrosis in the dominant (right) lung in our patient. Finally, because the physical and radiological findings indicated the laterality of chest wall mobility, dynamic breathing MRI was used to visualize the movement of the thorax, which showed significantly impaired mobility in the left side compared with the right; pleural adhesion and tracheal displacement after the previous lobectomy may have contributed to limited chest wall movement (21). Froese et al. recently revealed that mechanical tissue stretching leads to the development of pulmonary fibrosis, as it activates fibroblasts (22). In our patient, the greater chest wall mobility in the right lung may have stretched the tissue therein, thus precipitating unilateral ILD.

Based on the above, we believe that a combination of decreased perfusion, ventilation, and mobility of the left lung contributed to the asymmetrical interstitial pneumonia in our patient. Interestingly, one previous study has also reported on patients with unilateral pleuroparenchymal fibroelastosis (PPFE) (12). In that study, it was considered that impaired thoracic movement in the operated side triggered the development of unilateral upper lung field pulmonary fibrosis, especially in patients with an apical cap. However, in that study, all patients had lung lesions limited to the operated 


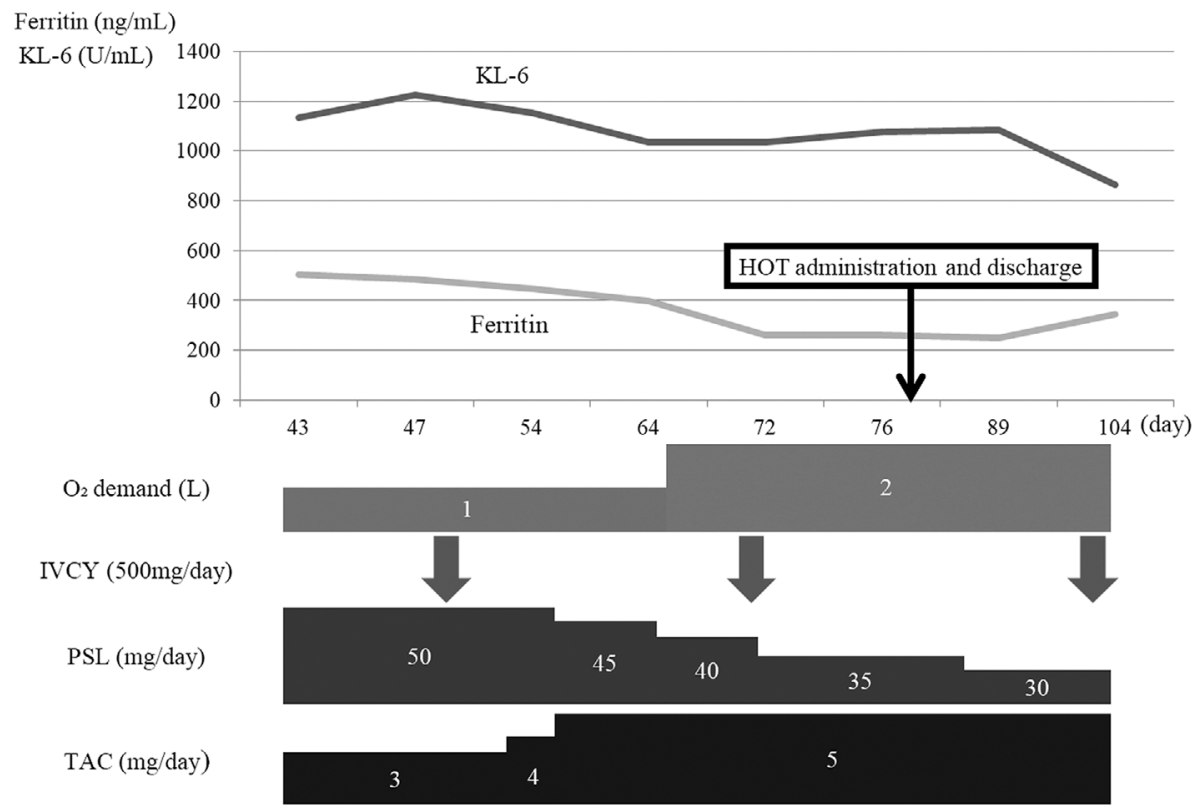

Figure 6. Clinical course of the patient. KL-6: Krebs von den Lungen-6, $\mathrm{O}_{2}$ : oxygen, IVCY: intravenous cyclophosphamide, PSL: prednisolone, TAC: tacrolimus

side, which is contrary to the presentation in our patient. Although it is possible that different pathogenic mechanisms will have divergent manifestations, further studies will be needed in order to obtain new findings concerning unilateral ILD.

\section{Conclusion}

We encountered a patient diagnosed with anti-MDA5 antibody-positive CADM who also developed asymmetrical ILD. The rapid diagnosis and treatment are essential for improving the prognosis in cases of CADM. In patients presenting with laterality of pulmonary findings, clinicians should be aware that atypical imaging findings may be observed.

The authors state that they have no Conflict of Interest (COI).

\section{Acknowledgement}

The authors greatly thank Dr. Mikiko Hiraoka, Department of Dermatology, Japanese Red Cross Medical Center, Tokyo, Japan, for her general support in the treatment of skin disease.

\section{References}

1. Callander J, Robson Y, Ingram J, Piguet V. Treatment of clinically amyopathic dermatomyositis in adults: a systematic review. $\mathrm{Br} \mathrm{J}$ Dermatol 179: 1248-1255, 2018.

2. Mukae H, Ishimoto H, Sakamoto N, et al. Clinical differences between interstitial lung disease associated with clinically amyopathic dermatomyositis and classic dermatomyositis. Chest 136: 1341-1347, 2009.

3. Ye $S$, Chen XX, Lu XY, et al. Adult clinically amyopathic dermatomyositis with rapid progressive interstitial lung disease: a retrospective cohort study. Clin Rheumatol 26: 1647-1654, 2007.

4. Tanizawa K, Handa T, Nakashima R, et al. HRCT features of in- terstitial lung disease in dermatomyositis with anti-CADM-140 antibody. Respir Med 105: 1380-1387, 2011.

5. Suga K, Tsukuda T, Awaya H, et al. Impaired respiratory mechanics in pulmonary emphysema: evaluation with dynamic breathing MRI. J Magn Reson Imaging 10: 510-520, 1999.

6. Troyanov Y, Targoff IN, Payette MP, et al. Redefining dermatomyositis: a description of new diagnostic criteria that differentiate pure dermatomyositis from overlap myositis with dermatomyositis features. Medicine (Baltimore) 93: 318-332, 2014.

7. Gerami P, Schope JM, McDonald L, Walling HW, Sontheimer RD. A systematic review of adult-onset clinically amyopathic dermatomyositis (dermatomyositis siné myositis): a missing link within the spectrum of the idiopathic inflammatory myopathies. $\mathbf{J}$ Am Acad Dermatol 54: 597-613, 2006.

8. Koga T, Fujikawa K, Horai Y, et al. The diagnostic utility of antimelanoma differentiation-associated gene 5 antibody testing for predicting the prognosis of Japanese patients with DM. Rheumatology (Oxford) 51: 1278-1284, 2012.

9. Moghadam-Kia S, Oddis CV, Sato S, Kuwana M, Aggarwal R. Anti-melanoma differentiation-associated Gene 5 is associated with rapidly progressive lung disease and poor survival in US patients with amyopathic and myopathic dermatomyositis. Arthritis Care Res (Hoboken) 68: 689-694, 2016.

10. Suzuki A, Kondoh Y, Taniguchi H, et al. Lung histopathological pattern in a survivor with rapidly progressive interstitial lung disease and anti-melanoma differentiation-associated gene 5 antibodypositive clinically amyopathic dermatomyositis. Respir Med Case Rep 19: 5-8, 2016.

11. Kuse N, Inomata M, Awano N, et al. Management and utility of transbronchial lung cryobiopsy in Japan. Respir Investig 57: 245251, 2019.

12. Sekine A, Satoh H, Iwasawa T, et al. Unilateral upper lung field pulmonary fibrosis radiologically consistent with pleuroparenchymal fibroelastosis after thoracotomy: a new disease entity related to thoracotomy. Respiration 94: 431-441, 2017.

13. Tcherakian C, Cottin V, Brillet PV, et al. Progression of idiopathic pulmonary fibrosis: lessons from asymmetrical disease. Thorax $\mathbf{6 6}$ : 226-231, 2011.

14. Tzouvelekis A, Anevlavis S, Bouros D. Angiogenesis in interstitial 
lung diseases: a pathogenetic hallmark or a bystander? Respir Res 7: 82,2006

15. Raghu G. The role of gastroesophageal reflux in idiopathic pulmonary fibrosis. Am J Med 115: 60S-64S, 2003.

16. Thomas A. Integrating mechanisms of pulmonary fibrosis. Wynn JEM 208: 1339, 2011.

17. Bast A, Weseler AR, Haenen GR, den Hartog GJ. Oxidative stress and antioxidants in interstitial lung disease. Curr Opin Pulm Med 16: 516-520, 2010.

18. Freeman B, Crapo J. Hyperoxia increases oxygen radical production in rat lungs and lung mitochondria. J Biol Chem 256: 1098610992, 1981.

19. Gerschman R, Gilbert BL, Nye SW. Oxygen poisoning and Xradiation: a mechanism in common. Science 119: 623-626, 1954.
20. Stevens J, Autor A. Proposed mechanism for neonatal rat tolerance to normobaric hyperoxia. Fed Proc 39: 3138-3143, 1980.

21. Lanza Fde C, de Camargo AA, Archija LR, Selman JP, Malaguti C, Dal Corso S. Chest wall mobility is related to respiratory muscle strength and lung volumes in healthy subjects. Respir Care 58: 2107-2112, 2013.

22. Froese AR, Shimbori C, Bellaye PS, et al. Stretch-induced activation of transforming growth factor- $\beta 1$ in pulmonary fibrosis. Am J Respir Crit Care Med 194: 84-96, 2016.

The Internal Medicine is an Open Access journal distributed under the Creative Commons Attribution-NonCommercial-NoDerivatives 4.0 International License. To view the details of this license, please visit (https://creativecommons.org/licenses/ by-nc-nd/4.0/).

(C) 2020 The Japanese Society of Internal Medicine Intern Med 59: 401-408, 2020 